\title{
Evaluation of an Automated CLIA System for the Determination of Carbohydrate Antigen 19-9
}

\author{
EMANUELA ANASTASI, CHANTAL DI SEGNI, SILVESTRA TUDINI, \\ GIUSEPPINA GENNARINI and ANTONIO ANGELONI
}

Department of Molecular Medicine, Policlinico Umberto I, Sapienza University of Rome, Rome, Italy

\begin{abstract}
Aim: To compare analytical characteristics between two different methods, a automated chemiluminescent immunoassay (CLIA) versus a radioimmunometric assay (RIA), for the determination of carbohydrate antigen 19-9 (CA19-9). Materials and Methods: One hundred and eighty-five blood samples from consecutively enrolled individuals ( 87 males and 98 females, aged 22-89 and 29-89 years, respectively) were evaluated. For both assays, a cut-off of $37 \mathrm{U} / \mathrm{ml}$ was used. Results: Comparison between assays was analyzed using Passing-Bablok regression, which showed a high inter-assay correlation ( $r=0.926)$. The mean intra-assay coefficient of variation was $17.6 \%$ (range $=6.1-50.7 \%)$ for the RIA $(n=10$ samples) and 5.2\% (range=0.4-17.0\%) for the CLIA assay ( $n=26$ samples). Conclusion: This study showed that CLIA for CA19-9 has a good reliability on all samples analyzed and should be preferred to RIA with the aim of reducing costs, greater standardization and more harmonized results.
\end{abstract}

Carbohydrate antigen 19-9 (CA19-9) (also known as cancer antigen 19-9, and sialylated Lewis (a) antigen), is a mucintype pentasaccharide which is specifically recognized by a specific monoclonal antibody (1). CA19-9 is the most frequently used tumor marker for cancer of the digestive tract, after carcinoembryonic antigen $(2,3)$. It is currently used in pre-treatment evaluation and in surveillance after treatment (4), both surgical and pharmacological, to early detect relapse or metastasis. At the present time, the use of CA19-9 for screening is not recommended due to several interferences that limit the specificity and sensitivity of the test. An increase of

Correspondence to: Emanuela Anastasi, Department of Molecular Medicine, University Sapienza, Viale Regina Elena 324, 00161 Rome, Italy. Tel: +39 064472347, Fax: +39 064478381, e-mail: emanuela.anastasi@uniroma1.it

Key Words: Tumor marker, carbohydrate antigen 19-9 (CA19-9), radioimmunometric assay (RIA), automated chemiluminescent immunoassay (CLIA). circulating level of CA19-9 not related to cancer arises in two main situations: i) inflammatory diseases of digestive tract, liver diseases and cholestasis, and ii) false positivity related to other possible interferences (5). Furthermore, CA19-9 is biochemically related to the Lewis A (BG-5, Le ${ }^{a}$ ) blood group substance; approximately $5-7 \%$ of all humans are genetically negative for Lewis blood group antigens and will be unable to produce the CA19-9 antigen even in the presence of malignant tissue (6). Phenotyping for the presence of the Lewis blood group antigen may be insufficient to detect true Lewis antigennegative individuals. Even patients who have Lewis antigenpositive genotype may produce varying levels of CA19-9 as a result of the gene-dosage effect.

In neoplastic pathology, CA19-9 level is useful not only when following-up patients with digestive tract tumors, but also for those with bile duct cancer, ovarian mucinous cystadenocarcinoma and uterine adenocarcinomas. A low CA19-9 level does not exclude the possible presence of a malignant tumor, just as a high level is not indicative of the existence of such tumor.

CA19-9 is measured by different methods such as immunoassays, immunoradiometric assays and automated immunometric assays employing, for example, chemiluminescent detection; the method actually in use at our laboratory is a manual radioimmunoassay (RIA) (CisBio ELSA-CA19-9; CisBio Bioassays, Codolet, France).

Various studies have shown the lack of harmonization of the results obtained with different methods, mainly because different molecular variant of this marker exist; furthermore the evaluation of CA19-9 performed with assays from different manufacturers can vary, due to differences in assay methods, antibodies used and reagent specificity (7). Hence with the aim of matching the performance and the reproducibility of two different methods used for the evaluation of CA19-9, we compared the RIA method (ELSA-CA19-9, CisBio) already in use in our laboratory and an automated analyzer based on chemiluminescence immunoassay (CLIA) (Snibe MAGLUMI CA19-9; Snibe Co., Ltd., Shenzen, China), independently of the clinical condition of patients. 

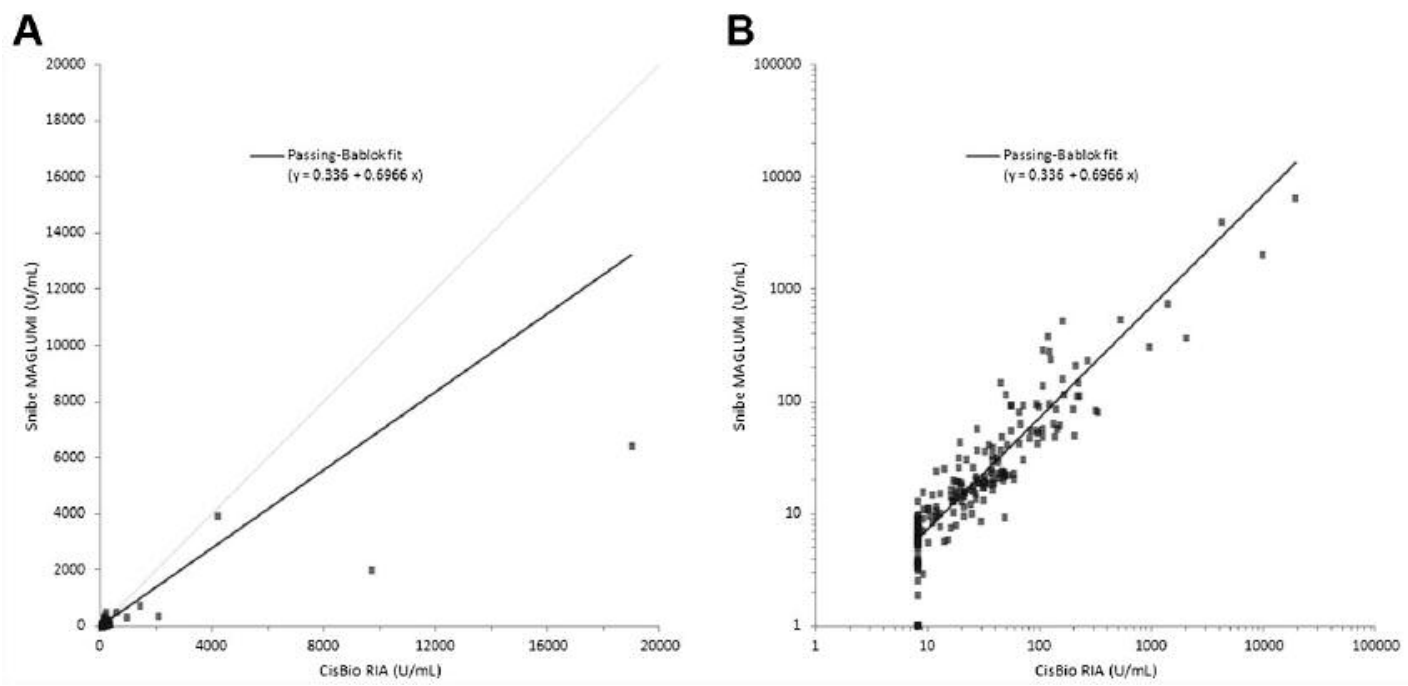

Figure 1. Comparison of the assays performed using Snibe's MAGLUMI and our reference radioimmunometric assay method (CisBio RIA) for the determination of carbohydrate antigen 19-9. Passing-Bablok regression plotted using linear (A) and logarithmic (B) scales.

\section{Materials and Methods}

After obtaining written informed consent, blood samples from 185 individuals (87 males and 98 females, aged 22-89 and 29-89 years, respectively) affected by oncological and non-oncological pathologies, enrolled consecutively from December 2015 to March 2016 were evaluated. All blood samples were sent to the Laboratory of Tumor Markers (Policlinico Umberto I, Sapienza University of Rome). All sera were obtained following a standard collection protocol: samples were collected in a Red Top Vacutainer, and centrifuged for $15 \mathrm{~min}$ at $3500 \times g$. The serum fractions were aliquoted and stored at $-80^{\circ} \mathrm{C}$ until analysis. Samples were thawed only once.

The level of CA19-9 were measured both by an RIA method (CisBio ELSA-CA19-9) and by an automated analyzer based on CLIA (Snibe MAGLUMI CA19-9). All assays were performed according to the manufacturers' instructions and both used a cut-off of normality of $37 \mathrm{U} / \mathrm{mL}$ based on the $95 \%$ confidence interval.

The RIA ELSA-CA19-9 assay is a solid-phase two-step sandwich immunometric assay in which monoclonal antibody to CA19-9 is coated on the ELSA (a solid phase fixed on the bottom of the tube) and another antibody to CA19-9 radiolabelled with iodine-125 is used as a tracer. The radioactivity bound to the ELSA is proportional to the amount of CA19-9 present in the sample at the beginning of the assay.

We compared this method with a fully-automated two-step sandwich CLIA (Snibe MAGLUMI CA19-9) which uses ABEI (is a nonenzymatic molecule with special structure to enhance acid and alkaline stability) to label an monoclonal antibody to CA19-9, and another antimonoclonal antibody to CA19-9 to coat magnetic microbeads. After a flash chemiluminescent reaction, the light signal is measured by a photomultiplier within $3 \mathrm{~s}$ (as relative light units; RLU) and is proportional to the concentration of CA19-9 present in the sample.

Statistical analysis. All analyses were performed using Analyse-It v.4.51 statistical software (Analyse-it Software, Ltd. Leeds, UK).

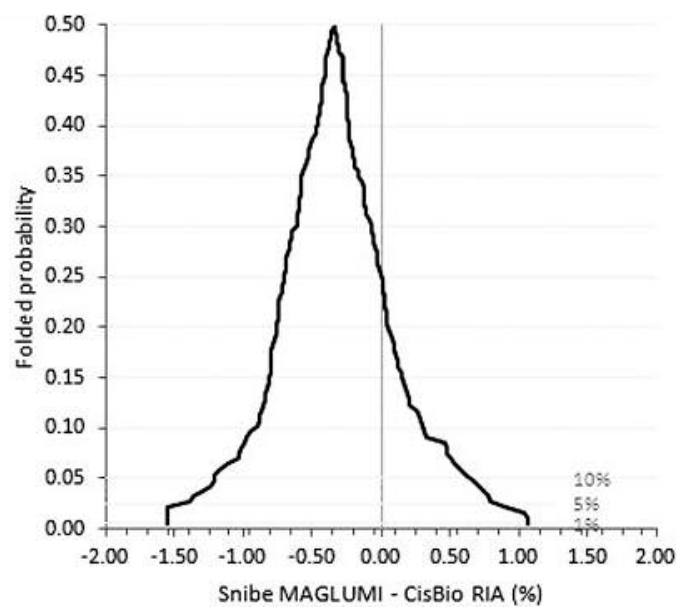

Figure 2. Mountain plot showing the distribution of differences in measurement between the chemiluminescent MAGLUMI immunoassay and radioimmunometric assay (RIA) methods for the determination of carbohydrate antigen 19-9, with an emphasis on the center and the tails of the distribution.

Comparison between assays was analyzed using Passing-Bablok regression, which is a robust, non-parametric statistical method which is not sensitive to outliers or the distribution of errors (8-10). Comparison studies were also performed evaluating the mountain plot, which shows the distribution of the differences with an emphasis on the center and the tails of the distribution (11-13), and using the BlandAltman method for difference plots, which shows the difference in measurement between two methods, allowing estimates of bias, with corresponding confidence interval (CI) (14-16). 

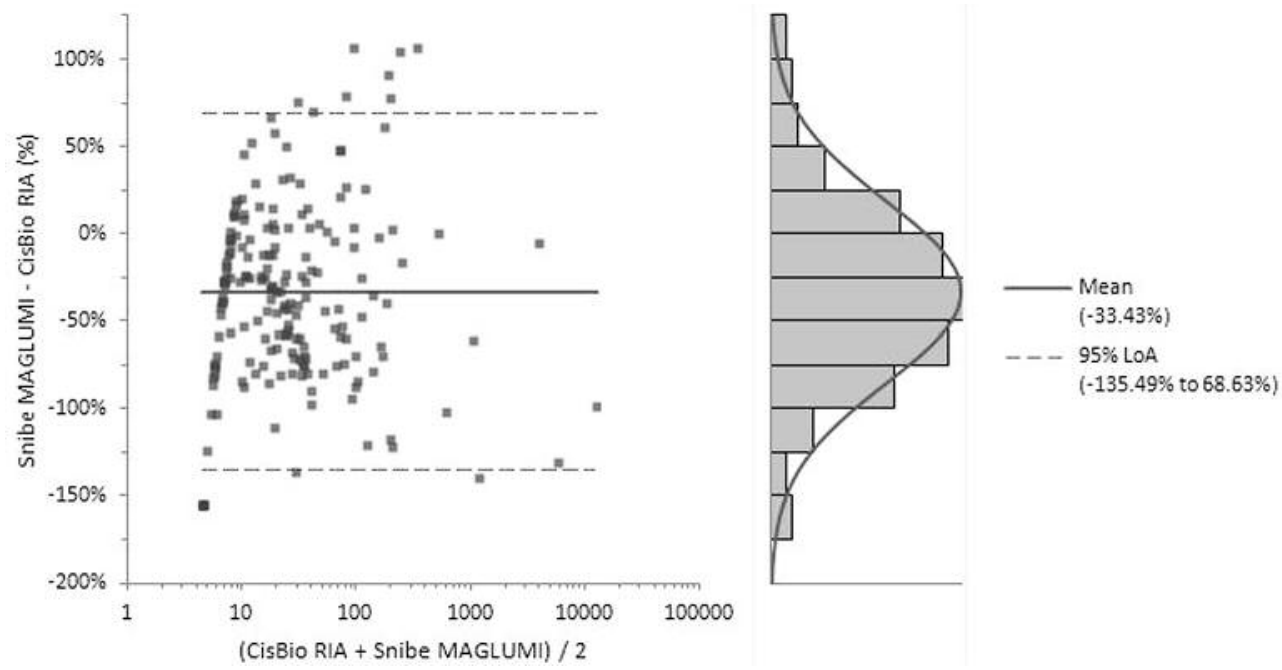

Figure 3. Bland-Altman difference plot of the relative differences between the chemiluminescent MAGLUMI immunoassay and radioimmunometric assay (CisBio RIA) methods for the determination of carbohydrate antigen 19-9 (CA19-9). The y axis shows the difference between the two paired measurements and the $x$ axis represents the average of these measures, as the best estimate of the true value. This plot is useful when the methods exhibit variability related to increasing magnitude, where the points on a difference plot form a band which starts narrow and becomes wider as $x$ increases to avoid an artificial relationship between the difference and magnitude (16). LoA: Limit of agreement, for comparison of the likely differences between individual results measured by the two methods.

A

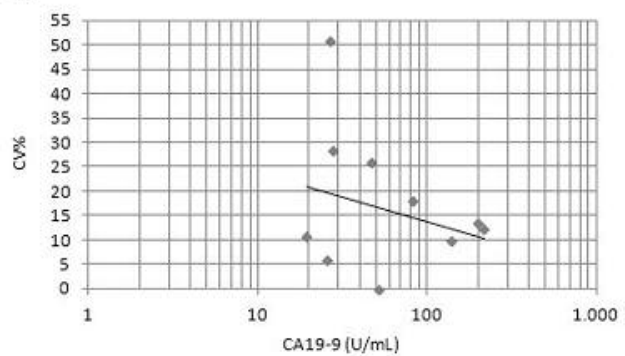

B

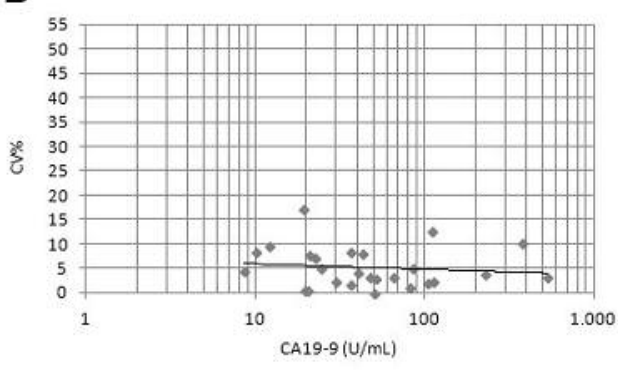

Figure 4. Precision of radioimmunometric assay (CisBio RIA) (A) and the chemiluminescent MAGLUMI immunoassay (B) for the determination of carbohydrate antigen 19-9 (CA19-9).

\section{Results}

The values for CA19-9 ranged from $8.00-18980 \mathrm{U} / \mathrm{mL}$ as determined by CisBio RIA and $1.00-6,435 \mathrm{U} / \mathrm{mL}$ as determined by Snibe MAGLUMI. Comparison of the assays performed using Snibe's MAGLUMI CA19-9 and our reference method showed a high interassay correlation [r=0.926, with slope of $0.6966(95 \% \mathrm{CI}=0.5730-0.8716)$ and intercept of 0.3360 (95\% CI=-2.271-2.027), CI based on 999 bootstrap samples] (Figure 1).

The results evaluated with mountain plot (Figure 2) and Bland-Altman difference plot showed a mean difference of $-33.43 \%$ (95\% limit of agreement=-135.49-68.63\%) (Figure 3).
The precision of MAGLUMI vs. RIA was determined by testing 26 and 10 samples, respectively, in duplicate. The mean intra-assay coefficient of variation (CV) was $17.6 \%$ (range $=6.1-50.7 \%)$ with RIA $(n=10$ samples) and $5.2 \%$ (range $=0.4-17.0 \%)$ with MAGLUMI assay $(\mathrm{n}=26$ samples) as shown in Figure 4.

The precision and linearity of MAGLUMI assay were also determined by testing serum pools from samples routinely referred to our laboratory, the results are expressed as CV. The intra-assay precision was tested in five replicates for three serum pools: low, intermediate and high CA19-9. The mean results were $8.9 \mathrm{U} / \mathrm{mL}(\mathrm{CV}=5.2 \%), 65.8 \mathrm{U} / \mathrm{mL}$ $(\mathrm{CV}=6.4 \%)$ and $206.6 \mathrm{U} / \mathrm{mL}(\mathrm{CV}=7.2 \%)$, respectively. 
The inter-assay precision for MAGLUMI was obtained with three serum pool replicates (low, intermediate and high CA19-9) in four different runs. The mean results were $9 \mathrm{U} / \mathrm{mL}(\mathrm{CV}=6 \%), 65.5 \mathrm{U} / \mathrm{mL}(\mathrm{CV}=5.6 \%)$ and $212 \mathrm{U} / \mathrm{mL}$ $(\mathrm{CV}=12 \%)$, respectively.

The inter-assay linearity was obtained by testing three dilutions in three replicates of a serum pool with a high CA19-9 value from samples routinely referred to our laboratory. Dilution factors used were 1:2, 1:10 and 1:100. The mean values were $179 \mathrm{U} / \mathrm{mL}(\mathrm{CV}=5.2 \%), 135.4 \mathrm{U} / \mathrm{mL}$ $(\mathrm{CV}=7.4 \%)$ and $256.0 \mathrm{U} / \mathrm{mL}(\mathrm{CV}=22.9 \%)$, respectively.

The inter-assay linearity of serum dilutions were tested in four replicates and mean values were $190 \mathrm{U} / \mathrm{mL}(\mathrm{CV}=6.6 \%)$ for the $1: 2$ dilution, $135.5 \mathrm{U} / \mathrm{mL}(\mathrm{CV}=3.3 \%)$ for the $1: 10$ dilution and $237.3 \mathrm{U} / \mathrm{mL}(\mathrm{CV}=22.2 \%)$ for the $1: 100$ dilution.

Both precision and linearity analyses showed that Snibe's MAGLUMI CA19-9 has good reliability as an alternative to RIA.

\section{Discussion}

CA19-9 was discovered in patients with cancer of the gastrointestinal tract (gastric, pancreatic and colon rectal cancer), bile duct cancer, ovarian mucinous cystoadenoma and uterine adenocarcinoma. This marker is very important for the management of oncological patients, especially before and after treatment, both surgical and pharmacological, and can provide important information on eventual relapse of disease or presence of metastasis.

The first method used to test CA19-9 was RIA through the use of monoclonal antibodies (1116NS19-9) (5). There are many variables that can lead to loss of linearity and correlation of RIA: the operator, dilution and incubation times used, and reaction kinetics. Nowadays, there are numerous automated assays which have replaced the RIA but they differ significantly in analytical performances, leading to an improvement in precision but worsening in the correlation between CA19-9 values obtained with different methods. We observed conflicting data in the CA19-9 results through the evaluation of External Quality Assessment between laboratories involved in the program.

The assays for the detection of CA19-9 and comparison between them have been studied extensively (7, 17-19), but according to Passerini et al. (17), interchangeability between methods for the evaluation of CA19.9 has not yet been achieved. For example Passerini and colleagues compared two different automated immunoassays for the determination of CA19-9, but despite their comparable diagnostic accuracy, the methods were not interchangeable (20); Stern et al. compared six different immunoassays for CA19-9 and found significant differences among systems (21).

Different causes may be involved in this lack of harmonization between the results obtained from different assays, for example, the lack of an international reference standard for use by the different manufacturers to appropriately calibrate their kits (17).

In this study, we evaluated MAGLUMI CA19-9 (Snibe Co., Ltd., Shenzen, China), an automatic assay system, for correlation, precision and analytical concordance with our reference method. Our comparison study performed with Passing and Bablok regression showed good agreement between MAGLUMI and RIA for the determination of CA19-9 ( $r=0.926)$. We also observed a good intra- and interassay precision for MAGLUMI CA19-9 assay.

This study showed that MAGLUMI CA19-9 allows better standardization and has a good reliability on all samples; moreover it enables testing of a greater number of samples with smaller amounts of reagents and produces more rapid results, with obvious clinical implications for the patients. In conclusion, MAGLUMI CA19-9 assay should be preferred to RIA with the aim of reducing costs and obtaining more rapid and standardized results in a shorter time.

\section{Acknowledgements}

This research did not receive any specific grant from funding agencies in the public, commercial, or not-for-profit sectors. The Authors are thankful to Valentina Viggiani, and Barbara Colaprisca for their technical assistance, and to Dr. Sara Tartaglione and Dr. Daniel Kenton for their contribution to English revision.

\section{References}

1 Betchel B, Wand A, Wroblewski K, Koprowski H and Thurin J: Conformational analysis of the tumor-associated carbohydrate antigen 19-9 and its LEA blood group antigen component as related to the specificity of monoclonal antibody CO19-9. J Biol Chem 265(4): 2028-2037, 1990.

2 Steinberg W: The clinical utility of the CA19-9 tumor-associated antigen. Am J Gastroenterol 85(4): 350-355, 1990.

3 Kim HJ, Kim MH, Myung SJ, Lim BC, Park ET, Yoo KS, Seo DW, Lee SK and Min YI: A new strategy for the application of CA19-9 in the differentiation of pancreaticobiliary cancer: analysis using a receiver operating characteristic curve. Am J Gastroenterol 94(7): 1941-1946, 1999.

4 Plebani M, Giacomini A, Beghi L, de Paoli M, Roveroni G, Galeotti F, Corsini A and Fraser CG: Serum tumor markers in monitoring patients: interpretation of results using analytical and biological variation. Anticancer Res 16(4B): 2249-2252,1996.

5 Galli C, Basso D and Plebani M: CA19-9: Handle with care. Clin Chem Lab Med 51(7): 1369-1383, 2013.

6 Vestergaard EM, Hein HO, Meyer H, Grunnet N, Jørgensen J, Wolf $\mathrm{H}$ and Ørntoft TF: Reference values and biological variation for tumor marker CA19-9 in serum for different Lewis and secretor genotypes and evaluation of secretor and Lewis genotyping in a Caucasian population. Clin Chem 45(1): 54-61, 1999.

7 La'ulu SL and Roberts WL: Performance characteristics of five automated CA19-9 assays. Am J Clin Pathol 127(3): 436-440, 2007. 
8 Passing $\mathrm{H}$ and Bablok W: A new biometrical procedure for testing the equality of measuremments from two different analytical methods. Application of linear regression procedures for method comparison studies in Clinical Chemistry, Part 1. J Clin Chem Clin Biochem 21(11): 709-720,1983.

9 Passing $\mathrm{H}$ and Bablok W: Comparison of several regression procedures for method comparison studies and determination of sample sizes. Application of linear regression procedures for method comparison studies in Clinical Chemistry, Part II. J Clin Chem Clin Biochem 22(6): 431-445, 1984.

10 Bablok W, Passing H, Bender R and Schneider B: A general regression procedure for method transformation. Application of linear regression procedures for method comparison studies in clinical chemistry, Part III. J Clin Chem Clin Biochem 26(11): 783-790, 1988.

11 Krouwer JS and Monti KL: A simple, graphical method to evaluate laboratory assays. Eur J Clin Chem Clin Biochem 33(8): 525-527, 1995.

12 Monti KL: Folded empirical distribution function curves mountain plots. Am Stat 49(4): 342-345, 1995.

13 Xue J-H and Titterington DM: The p-folded cumulative distribution function and the mean absolute deviation from the p-quantile. Stat Probab Lett 81(8): 1179-1182, 2011.

14 Altman DG and Bland JM: Measurement in medicine: the analysis of method comparison studies. Statistician 32(July 1981): 307-317, 1983.

15 Bland JM and Altman DG: Statistical methods for assessing agreement between two methods of clinical measurement. Lancet 327(8476): 307-310, 1986.

16 Bland JM and Altman DG: Measuring agreement in method comparison studies. Stat Methods Med Res 8(2): 135-160, 1999.
17 Passerini R, Riggio D, Salvatici M, Zorzino L, Radice D and Sandri MT: Interchangeability of measurements of CA19-9 in serum with four frequently used assays: An update. Clin Chem Lab Med 45(1): 100-104, 2007.

18 Hotakainen K, Tanner P, Alfthan H, Haglund C and Stenman UH: Comparison of three immunoassays for CA19-9. Clin Chim Acta 400(1-2): 123-127, 2009.

19 Deinzer M, Faissner R, Metzger T, Kaminski, Wolfgang E, Löhr M, Neumaier M and Brinkmann T: Comparison of two different methods for CA19-9 antigen determination. Clin Lab 56(7-8): 319-325, 2010.

20 Passerini R, Cassatella MC, Boveri S, Salvatici M, Radice D, Zorzino L, Galli C and Sandri MT: The pitfalls of CA19-9: Routine testing and comparison of two automated immunoassays in a reference oncology center. Am J Clin Pathol 138(2): 281287, 2012.

21 Stern P, Friedecky B, Bartos V, Bezdickova, D, Vavrova,J, Uhrova J, Rozprimova L, Zima T and Palicka V: Comparison of different immunoassays for CA19-9. Clin Chem Lab Med 39(12): 1278-1282, 2001.
Received August 9, 2016

Revised September 15, 2016

Accepted September 16, 2016 